\title{
A MOBILE HOSPITAL - ITS ADVANTAGES AND FUNCTIONAL LIMITATIONS
}

\author{
J. BAKOWSKI \\ Faculty of Architecture, Gdansk University of Technology, Poland.
}

\begin{abstract}
The idea of a mobile hospital is not entirely new - the need for their use, ranging from military field hospitals to the charity or mass disaster tent cities, shows how much they are needed. While these cases can be reduced to a portable ambulatory unit with a limited scope of outpatient medical activities, a fully functional hospital is a system with a high degree of complexity far exceeding a regime of compact objects.

Solutions belonging to the category of mobile architecture are increasingly common, technical and logistic difficulties are solved in a more perfect way. This applies mostly to objects with simple layouts and functional relationships, with relatively little technical equipment. In the case of healthcare facilities there are several factors increasing the difficulty: multitude of functional connections, sanitary requirements, technical equipment, and internal infrastructure. This raises the question if it is possible to build a hospital as a modular and mobile structure.

An emergency department (ED), regardless of principles of its operation, is a fully independent unit of a hospital. Depending on the solutions it can operate as a stand-alone entity (as in the American solutions), or in conjunction with the whole hospital (but still autonomously, as in European solutions). Taking some simplifications the ED can be treated as a miniature hospital, in its internal structure one can find all the elements that make up a fully functional hospital and the scale of solutions allows enclosing the ED layout on a relatively small area.

Construction of a mobile hospital unit is a task of interest from different points of view: the applicability of this approach includes both the military and civilian objectives. The paper tries to answer the question what terms and conditions must meet the functional system of such a healthcare facility, especially what are the boundaries for the fully functioning emergency mobile hospital. Strictly architectural issues, i.e. the form or construction and materials used to build such structures are out of scope of this paper.
\end{abstract}

Keywords: emergency hospital, healthcare architecture, mobile hospital.

\section{INTRODUCTION}

Who and to what extent needs a mobile hospital? An answer to this question is relatively simple: there is a demand for a hospital everywhere, where an access to a traditional, stationary health care facility is limited. A deeper development of this sentence can introduce a more detailed distinction in ways of organisation and functioning of a hospital. Then, what are the possible fields of operation for the mobile hospital? Why is this distinction significant? [1].

The easiest way to answer this question is to point to the balance between the needs and possibilities: a mobile hospital, despite the fact that it is in compliance with the tasks of primary health care, thanks to its organizational form and technological requirements, is a part of specialist medical care. The effort needed to organize and run such a hospital far exceeds the expenditure necessary to run the stationary hospital $[1,2]$.

The elementary difference concerns the separation of "treatment" (primary health care, including health promotion and preventive activities) and "emergency" (acute and crisis situations) activities. An emergency medicine, no matter of the form of its organisation, is a set of medical actions, serving a life rescue. Its range consists of any medical procedures associated with sudden injuries or trauma. The understanding of mobile hospital leads to an idea of 
military hospitals or to hospitals of reacting to disasters, both natural and those resulting from human factor. Following this way of thinking, the function of mobile hospital can be confined and compared to the area of operation of emergency department (ED). Certainly, such "department" should be fully equipped in the terms of technical, technological and treatment (surgical and diagnosis) infrastructure required for its proper functioning, but still its essence is the same as an ED - it is an immediate reaction to the state of threat of health or life which leads to a removal of that threat and at this point, theoretically, its role ends itself. Hospitalisation, covering patients' convalescence, is not included in the range of actions of such a facility.

The matter of "therapeutic" hospitals is a completely different issue. In this case a mobile hospital is a facility entirely substituting functions of stationary hospitals. A hospital, to be assigned as a rightful element of the health care system, has to consist of almost every department: diagnostics, treatments or bed wards. Their mutual relations and the principal of functional and spatial separation of each department cause a stepped complication of a hospital's layout. It becomes a portable rather than mobile object: designing and construction of many distinct modules and their further transport eliminates a hospital from quick and interim use. An advantage of the solution can be taken in situation of the expected longer period of action, for example, as a hospital for humanitarian missions on areas deprived of easy and rapid access to the health care system or as a replacement hospital in case of stationary hospital being unable to fulfil its function (for instance during its extension or renovation).

The article is an attempt to answer consecutive questions: what is the purpose of mobile hospitals? How can it be realised?

\section{CRITERIA OF MOBILE HOSPITALS' EFFICIENCY AND FUNCTIONING}

A response to these questions requires several criteria assuming and evaluating accepted solutions to be established. These criteria can be divided into two groups:

1. Construction and logistic criteria.

2. Functional and technological criteria.

First criterion involves technical solutions, related not only to problems of construction of the mobile hospital unit, but to the mobile architecture generally. These issues can be brought to 2 terms: modularity (an ability to pack the function into a cubic container) and mobility (understood as an easiness of the construction to be transported from one to other place). Modularity should provide a construction of possibly most numerous and diverse structures of various purposes. Mobility is to ensure a solution which enables the module to be loaded and transported by standard ways of shipping. In this way a construction module of mobile architecture approaches construction and size of the typical commodity container, transported either by a truck or a ship, or even air-borne.

A construction module is at the same time an advantage and a disadvantage of mobile hospital solution. On the one hand a spatial module is easy in production and transport; on the other hand its dimensions are not suited properly to the needs of medical technology, especially on the level of building layout arrangements containing several rooms of different purpose. From the ergonomic point of view, a problem lies in the floor plan of the module: the shape of elongated rectangle which imposes linear solutions of functional layout. Moreover, a height of standard container is insufficient to use advanced installation systems, 
especially in terms of heating, ventilation and air-conditioning (HVAC). In particular equipping an operating theatre with laminar airflow requires large spaces for the installation systems to be spread [3].

Functional criteria result partially from the problems mentioned above. Regardless of the mobile hospital type, general rules of health care facilities design must be respected. These are first of all: a principle of isolating functional units, rigorously obeyed aseptic and ergonomic issues in accordance with detailed demands of medical procedures. From a spatial or architectural point of view, this fact translates into the use of complex functional layout, based on structural and installation grid (multispan grid, i.e. rectangular or other shape array of structural pillars connected to installation system), allowing to free arrangement of both larger hospital departments (or suits of rooms) and particular rooms within them. The peculiarity of mobile architecture excludes (or at least considerably limits) the solutions. In the case of stationary building the role of the frame holding the whole structure is performed by its internal system of communication (hospital "street"). In the case of mobile hospital, ensuring the proper solution requires quite a number of "empty" modules serving only communication purposes. From the economic point of view, this is inefficient, regarding the costs of transport of modules not serving the main task directly.

The technological criterion seems to be relatively simple to fulfil. And that is also in case of military or field hospitals. Simultaneously with the higher degree of spatial layout complexity, the installation systems become more and more compound, especially the infrastructure of the mechanical ventilation.

\section{MODULAR HIERARCHY}

Accepting these criteria one comes to another question: which module of mobile hospital is the most important one? Whether is it a treatment one? Diagnostics? or hospitalisation?

Before it is possible to answer this question, it is crucial to analyse one more difference between mobile and stationary hospital. Stationary hospital, like every building is a threedimensioned construction in every aspect. Also, its functional layout is built in three dimensions: its departments are located on various storeys, connected with complicated systems of internal communication, the vertical ones as well. In an obvious way, it makes rooms' separation and zoning possible, it organises the flow of patients, personnel and materials and it allows controlling the access to the each individual suite of rooms. In the case of mobile hospital, a temporary structure assembled from ready-to-use elements which is not permanently attached to the ground, the functional layout is simplified to the "flat", two-dimensional structure. Assembling functional structures is still possible, whether in the form of linear layouts (with sequences of the single room-modules) or areal, multispan ones (in a similar way to stationary hospital), enabling unrestricted functional solutions, suited to technology of medical procedures. With this assumption, a key role is performed by communication modules and installation solutions (which can also be based on uniformed modules).

Why is the communication module so important? In stationary hospital, the area of communication ranges from $20 \%$ to $30 \%$ of the total surface of the facility. It is a relatively significant number, comparing to $10 \%-20 \%$ in other types of buildings. An extensive area of corridors in health care facilities results from their peculiarity: there are many different groups of users; with strong hierarchisation of access to particular rooms or suits of rooms. A differentiated availability demands a specialised communication: general, soiled, clean or sterile corridors. The mobile hospital, in spite of its straightforwardness and obvious restrictions, also has to be suited to these requirements of asepsis and medical technology. 


\section{TECHNICAL EQUIPMENT}

Also in case of technical equipment there are two options to be chosen (correspondingly to the case of stationary hospital): technical supply in dispersed or centralised system. Regardless of the form of realisation, the basic task of the equipment is concentrated on energy supply (electric, heating), fresh water piping and sanitary, providing appropriate room ventilation and special convey (medical gases, vacuum).

Not every module requires supply with all the media and in the same amount. Clearly the most demanding modules are diagnostic and treatment ones, particularly the operating room. In the case of stationary hospital, especially when it has a separate technical floor over the most demanding departments, setting a room or a suite of rooms on the same floor is not a problem at all. The distribution of installation systems is so flexible that it allows the free layout of the rooms. Additionally, within the setting subsequent changes in the spatial and functional modifications or even complete change in use are acceptable (and even more, such changes are actually planned as a dynamic functional variation of every health care facility). Another problem is the exploitation of installation systems. Necessary service access to equipment and fittings, as well as their operation, excludes integrated solutions where system components are located directly in the treatment or diagnostic module [3].

So, mobile hospital, despite its functional simplicity (or because of it) lacks the distinctive feature of the stationary architecture: distribution system of installations. This absence enforces direct access to auxiliary (supply) modules in order to eliminate the entire distribution system throughout the facility. In this way, the modularity significantly reduces the freedom and setting of individual modules. Their spatial orientation is planned in advance and is not subject to subsequent modifications.

\section{SHELTER}

The external environment, regardless of the climatic conditions, for the hospital always is a hostile environment. Regardless infection control system used a hospital always has to be separated from the environment with a partition of high performance. From an architectural point of view, this shelter is obviously the most interesting part of the solution. The building itself and its interior play an important role in the healing process - properly designed creates an environment ready to support medical processes (healing environment).

Even though the new possibilities in the construction of the module itself, this topic goes far beyond the scope of this article. Literature provides many examples of unusual and innovative solutions [4]; many of them can be expanded and adapted to the needs of mobile hospitals.

\section{MODEL OF EMERGENCY MOBILE HOSPITAL}

A "military" or rather an "emergency" mobile hospital should be considered at first. With some simplification, it can be considered as a limited model of ED: it consists of basic diagnostic equipment and relatively simple treatment rooms, corresponding to an operation or the resuscitation room of ED. There are two variants of ED, taking into account organisation of space or technological line flow: American and European model [5]. They can be compared on a basis of the same sets of medical procedures (and hence, the same sets of modules) being conducted at each of the model (Fig. 1).

The difference between the two models comes down to one important aspect: in the European ED patients' flow is unidirectional, almost linear, from the entrance of the patient to the segregation area (TRIAGE), through diagnostic and treatment facilities ending at the hospital bed wards (this way the ED is only a method of hospitalization, not a place for it). This is due to the principles of ED operation. ED is an integral part of the hospital and at the same time 

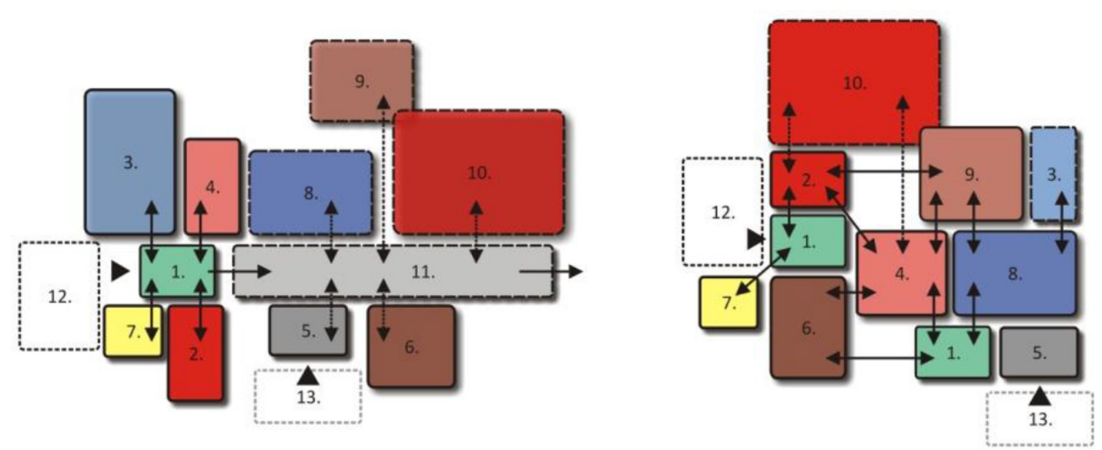

Figure 1: Emergency departments, diagrams according to a European (left) or American (right) or model. Key: 1-TRIAGE area, 2-trauma (resuscitation), 3-observation unit, 4-therapy/treatment, 5-waiting, 6-consultation, 7-registry/admission, 8intensivecare unit,9-laboratory/diagnostics, 10-operatingroom,11-communication, 12-ambulance entry, 13-pedestrian entry [5].

it is an emergency path of hospitalization (while the second option is a regular admission). The American ED is more autonomous unit, operating often as an outpatient clinic compared to European standards. In effect the TRIAGE and central nurse station become the most important areas of the ED (as the control point and distribution of all other activities). Other functions of the ED are grouped around the focal point of the nurse's station (or the point of care). The flow of patients through the ED is less structured, without clearly separate movement paths, always through the nurse's point.

To compare the possibilities of setting up a mobile hospital due to these two models there is a list of common facilities (Table 1) (Figs. 2 and 3). For analysis a standard 40' module has been taken; facilities area according to Polish standards [6].

There are numerous examples of mobile hospitals built as emergency hospitals [7-10]. Most of these products are flexible enough to meet specialized needs of any customer, including functional, environmental and supply needs, though in some cases, they are adjusted to

Table 1: List of the ED facilities.

\begin{tabular}{llccc}
\hline & & & \multicolumn{2}{c}{ No. of modules } \\
\cline { 4 - 5 } No. & Function & Area $\left[\mathrm{m}^{2}\right]$ & Eur. & Am. \\
\hline 1 & TRIAGE & 28 & 1 & 1 \\
2 & Trauma & 28 & 1 & 1 \\
3 & Observation & 14 & 2 & 3 \\
4 & Therapy/Treatment & 28 & 1 & 1 \\
5 & Consultation & 14 & 1 & 1 \\
6 & Registry/Admission & 28 & 1 & 1 \\
7 & Intensive care & 28 & 1 & 1 \\
8 & Laboratory/Diagnostics & 28 & 1 & 1 \\
9 & Operating suite & 28 & 1 & 1 \\
& Total & & 10 & 11 \\
\hline
\end{tabular}




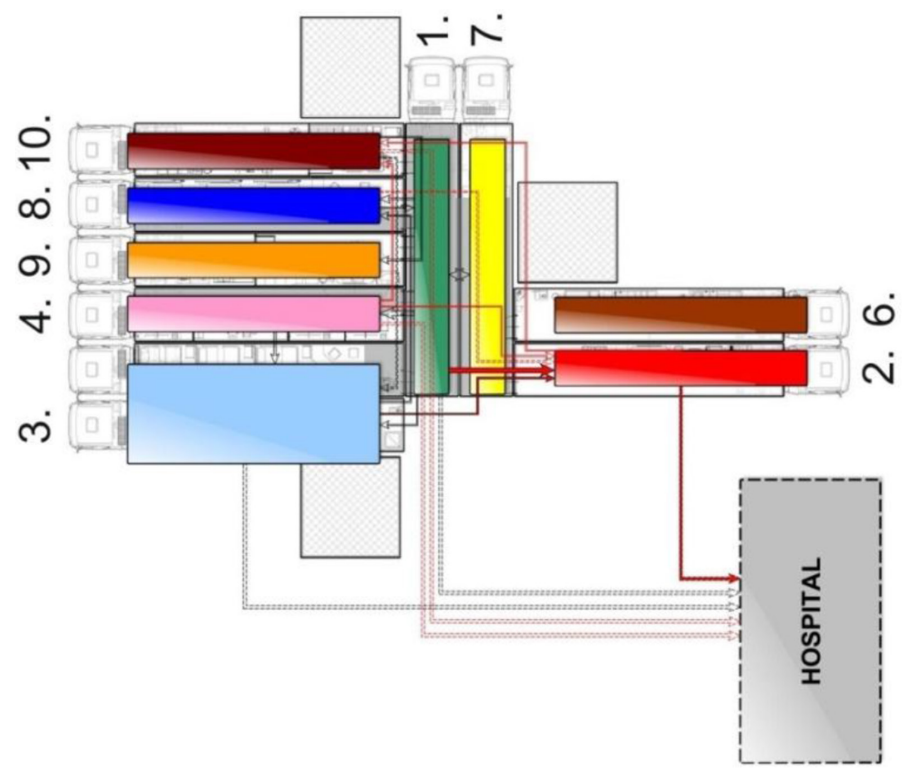

Figure 2: Emergency department, set up as a mobile hospital based on a typical truck size. Diagram according to a European model. Key as above.

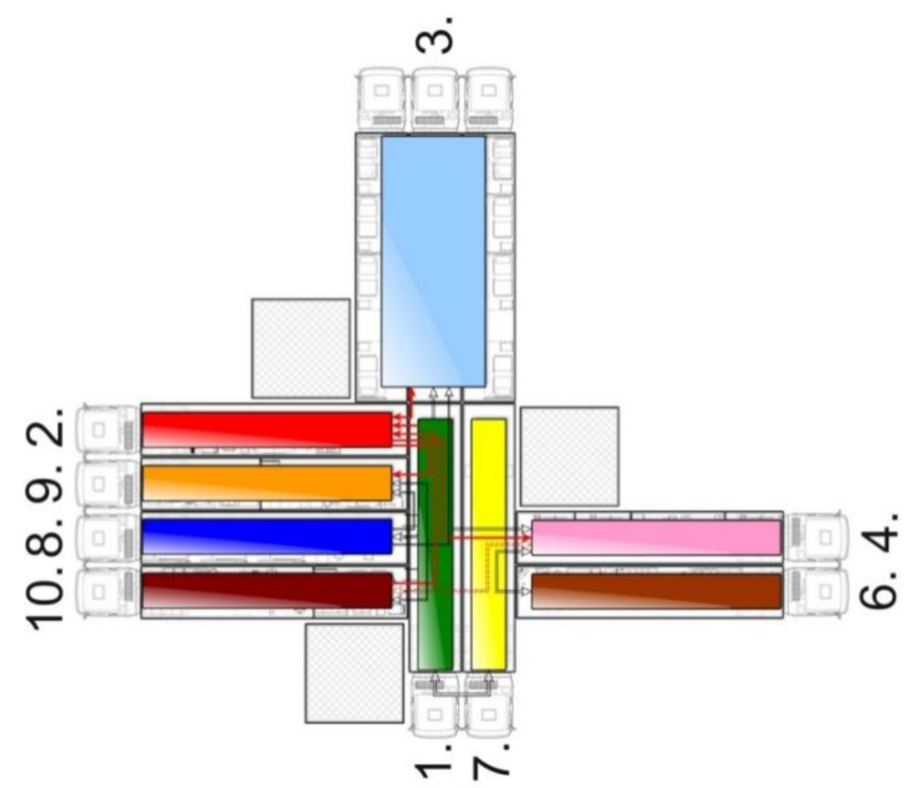

Figure 3: Emergency department, set up as a mobile hospital based on a typical truck size. Diagram according to an American model. Key as above. 
fulfil a specific role as a humanitarian facility. This restriction limits the scope of operation of these facilities as they lack some of the more advanced equipments or units.

\section{MODEL OF HEALING MOBILE HOSPITAL}

The model of "healing" hospital is to be distinguished by a much larger number of medical procedures, possible to carry out at this facility [11]. Still, they can be performed in a simplified manner, in conditions corresponding to the standards of mobile hospital, but their accumulation causes a significant increase in the complexity of the spatial arrangement of the facility. Model solution for the whole hospital is a risky, if not impossible task. Because of the complex interrelations between hospital departments finding a common scheme of functional layout is not possible (even in case simplified mobile hospital).

As with the emergency hospitals there are several ready-to-use solutions that can be considered as healing hospitals, or that can be easily transformed to this variant [8, 12-15].

\section{CONCLUSIONS}

The modular container is not the only possible solution, there could be many variants of different structures: tent or pneumatic structures and lightweight constructions [4]. Considering them as a shelter for any healthcare facility one can point out a considerable disadvantage; their purpose is to cover a single-use, homogeneous space, without any rigorous needs for dividing space into separate rooms. Thus, the modular systems with separate units insofar seem to be the optimal solution for the both types of mobile hospitals, as they can be easily and quickly assembled and disassembled, keeping their ability to being ready-to-use. On the other side, the modularity of the mobile hospital is not an inseparable issue of the mobile architecture idea. Maybe this paradigm is not that obvious? There are new ways of production and construction that can completely revolutionize the way we think about the portable architecture. An assumption that mobile or portable hospital is a lightweight, removable design for fast time could climb significantly change the way its operation - especially in the case of hospital treatment.

The models of mobile emergency hospital prove that it can be assembled with up to 10-11 building blocks, and using 6-8 variations of these modules (depending on the model of the ED). Samples included in the paper certainly are not the only possible; their goal was to prove how many units are needed to build a fully functioning ED facility (Figs. 4 and 5). Still, there are several problems to be considered and solved. The most important ones are to provide proper connections between various areas of the facility and to keep high standards for infection control. The latter, as a fundamental for any operational procedures requires the HVAC systems to be provided with additional solutions - for example with strict control of air-flow directions and pressures.

Considering mobile buildings, especially mobile hospitals, one may be tempted to check what rules of architecture are met. As the portable architecture still is the art of erection of fine buildings, the classic principles of architecture could be applied. As there are many historic and contemporary theories, they can be limited to the two original ones. In accordance with good architecture by Vitruvius, any edifice should implement the three rules [16]: durability, utility, beauty. Other, more contemporary rules of good architecture can be found in Le Corbusier works [17]: pilotis, free design of the ground plan, free design of the façade, horizontal window and roof gardens. Which of these rules relate to the mobile architecture, particularly to the mobile hospital architecture? Certainly Vitruvius' durability is not an especially valued hallmark, at least understood in the classical way. These structures are not being 


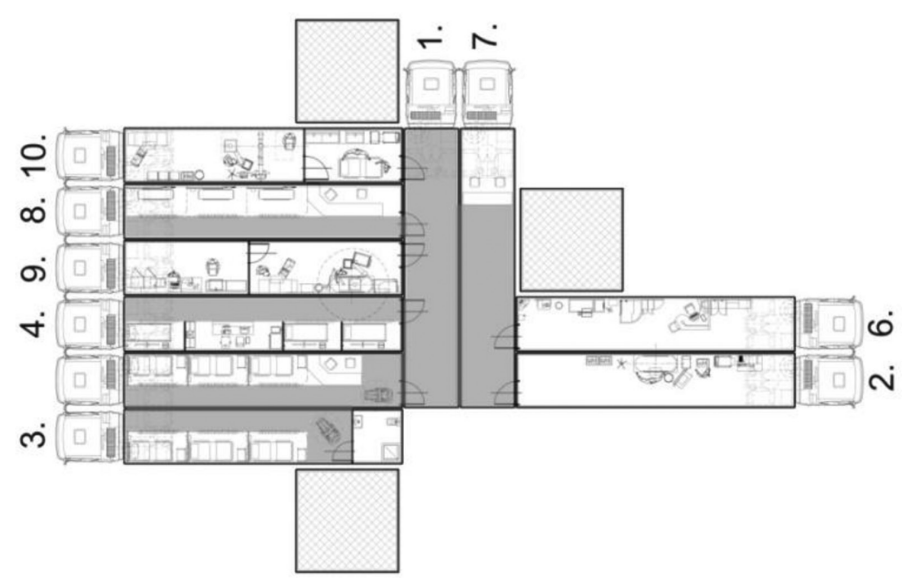

Figure 4: Mobile emergency hospital. Diagram showing basic equipment for required medical procedures, according to a European model. The inner communication shown in grey. Key as above.

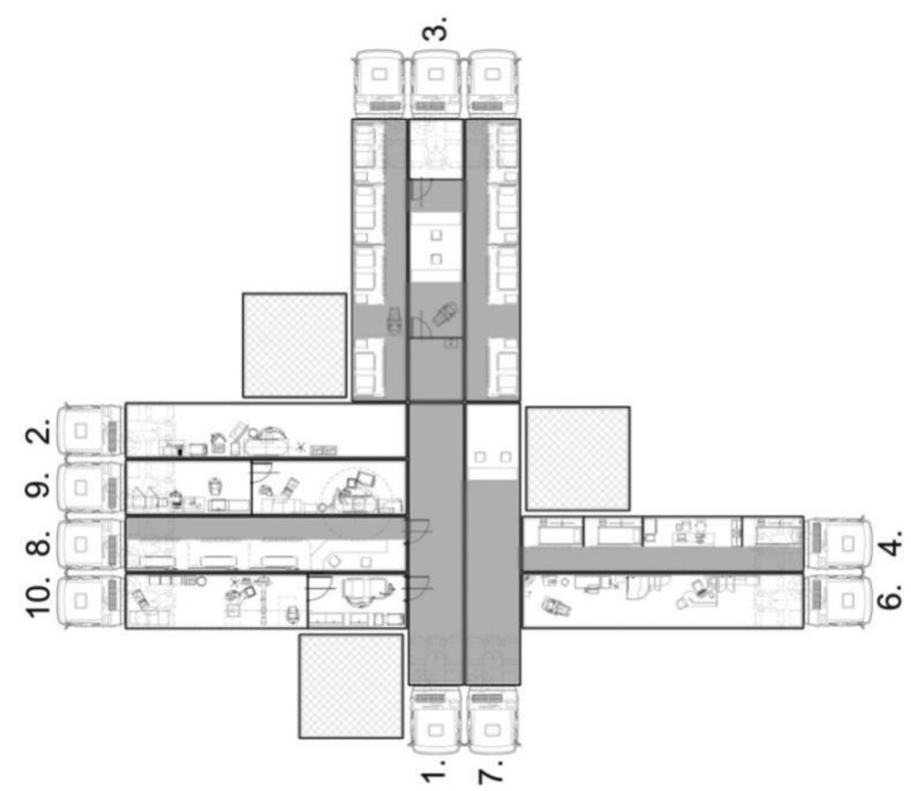

Figure 5: Mobile emergency hospital. Diagram showing basic equipment for required medical procedures, according to an American model. The inner communication shown in grey. Key as above.

constructed just to last; they are built for a specific and utilitarian purpose. Also aesthetic issues do not play a leading role, but the question is subjective. The modern approach requires an open floor plan to fit diverse activities; taking into account technological limitations this goal can be perfectly accomplished with modular units. Regardless of the criteria for the 
assessment of this architecture, a fundamental feature of mobile hospital quality is its usability and ease of moving it to another location.

\section{REFERENCES}

[1] International Committee of the Red Cross, Mobile Health Units. Methodological Approach, Geneva, Switzerland, 2006.

[2] Bricknell, M.C.M., The military approach to medical planning. Conflict and Catastrophe Medicine, ed A.H. Buma, Springer-Verlag: London Limited, pp. 177-187, 2009. http://dx.doi.org/10.1007/978-1-84800-352-1_11

[3] Kaiser, K. \& Wolski, A., Klimatyzacja $i$ wentylacja $w$ szpitalach. Teoria i praktyka eksploatacji, IPPU Masta Gdańsk, pp. 55-69, 2007.

[4] Kronenburg, R., Portable Architecture. Design and Technology, Birkhäuser: BaselBoston-Berlin, 2008.

[5] Bąkowski, J., Several notes on differences between American and European model of an emergency department. An architect's point of view. 1st Annual International Conference Health and Medical Sciences, Athens, Greece (p. 15). Athens Institute For Education and Research, Greece, 2013.

[6] Ustawa o działalności leczniczej, Dz. U. $2011 \mathrm{nr} 112$ poz. 654.

[7] CROSSMOBIL GmbH, available at: http://www.crossmobil.com/mobile-hospitals/ mobile-hospitals/the-mobile-emergency-hospital-intensive-care-version/

[8] ZEPPELIN MOBILE SYSTEME GmbH, available at: http://www.zeppelin-systeme. de/hospitalbmqe.htm

[9] METAL PROM EXPORT LLC, available at: http://mpe-export.com/mobile_hospital. html

[10] GAMA Holding, available at: http://www.gama.com.tr/en/gama-trading/products/ mobile-hospital-solutions/340

[11] Khanna, A.B. \& Narula, S.A., Mobile health units: mobilizing healthcare to reach unreachable. International Journal of Healthcare Management, 9(1), pp. 58-66, 2016. http://dx.doi.org/10.1080/20479700.2015.1101915

[12] ADVANCED MOBILE HOSPITAL SYSTEMS Inc, available at: http://www.amohs. com/main-gallery/

[13] MOBILE HEALTHCARE FACILITIES LLC, available at: http://www.mhcfac.com/ facilities/mobile-hospitals/

[14] NAFFCO FZCO, available at: http://www.naffco.com/products/view/mobile-fieldhospital

[15] GLOBAL OCEAN \& LAND DEVELOPERS INTERNATIONAL LLC, available at: http://goldev.net/portfolio-item/mobile-hospital/

[16] M. Vitruvius Pollio, De architectura, ca. 10 BC.

[17] Le Corbusier, Vers une architecture, 1920s. 